\title{
Quasifixed points from scalar sequestering and the little hierarchy problem in supersymmetry
}

\author{
Stephen P. Martin \\ Department of Physics, Northern Illinois University, DeKalb, Illinois 60115, USA
}

(Received 26 December 2017; published 9 February 2018)

\begin{abstract}
In supersymmetric models with scalar sequestering, superconformal strong dynamics in the hidden sector suppresses the low-energy couplings of mass dimension 2, compared to the squares of the dimension-1 parameters. Taking into account restrictions on the anomalous dimensions in superconformal theories, I point out that the interplay between the hidden and visible sector renormalizations gives rise to quasifixed point running for the supersymmetric Standard Model squared mass parameters, rather than driving them to 0 . The extent to which this dynamics can ameliorate the little hierarchy problem in supersymmetry is studied. Models of this type in which the gaugino masses do not unify are arguably more natural, and are certainly more likely to be accessible, eventually, to the Large Hadron Collider.
\end{abstract}

DOI: 10.1103/PhysRevD.97.035006

\section{INTRODUCTION}

Low-energy supersymmetry [1] has historically been one of the most well-studied solutions for the hierarchy problem associated with the electroweak scale. This popularity has been on the wane as the continuing explorations of the LHC have so far not found any evidence for the existence of superpartners. However, it is notable that no evidence for any of the other proposed solutions of the hierarchy problem has been found either; LHC searches for new physics have not produced any enduring positive signals. This state of affairs suggests that, regardless of the fate of supersymmetry, some new idea might be needed in order to understand the small size of the electroweak scale.

Within the context of supersymmetry, the problem is sometimes called the "little hierarchy problem" and can be illustrated with an equation that relates electroweak symmetry breaking to the parameters of the minimal supersymmetric Standard Model (MSSM) Lagrangian:

$-\frac{1}{2} m_{Z}^{2}=m_{H_{u}}^{2}+|\mu|^{2}+\frac{1}{2 v_{u}} \frac{\partial(\Delta V)}{\partial v_{u}}+\mathcal{O}\left(1 / \tan ^{2} \beta\right)$.

This equation follows from minimizing the Higgs potential and relates the $Z$ boson mass to the supersymmetrypreserving and -breaking Higgs squared masses $|\mu|^{2}$ and $m_{H_{u}}^{2}$ (in the notation of Ref. [1]) and the loop-suppressed

Published by the American Physical Society under the terms of the Creative Commons Attribution 4.0 International license. Further distribution of this work must maintain attribution to the author(s) and the published article's title, journal citation, and DOI. Funded by SCOAP ${ }^{3}$. corrections to the effective potential, $\Delta V$, which depends on a vacuum expectation value $v_{u}$ for the Higgs field that couples to the up-type quarks and squarks. These loop corrections can be made small by an appropriate choice of renormalization scale, typically of order the geometric mean of the top-squark masses. The remaining tree-level and loop contributions in Eq. (1.1), suppressed by $1 / \tan ^{2} \beta$ for large $\tan \beta$, are small enough to be neglected in a first approximation, if $\tan \beta$ is big as indicated by the observed Higgs scalar boson mass of $125 \mathrm{GeV}$. The little hierarchy problem is that in the MSSM, boundary conditions and radiative corrections correlate $m_{H_{u}}^{2}$ to the mass scales of the superpartners that now seem to be much heavier than $m_{Z}$, with lower mass bounds that continue to rise with each new reported LHC search, and top-squark and other superpartner masses well above $1 \mathrm{TeV}$ also favored independently by $M_{h}=125 \mathrm{GeV}$.

There is no hierarchy problem associated with $\mu$, which is a superpotential parameter and therefore protected by a chiral symmetry; the smallness of its magnitude compared to any larger mass scale is technically natural. This has led to considerations of "natural supersymmetry" scenarios, which in general suppose that somehow (with the complete explanation perhaps postponed) $m_{H_{u}}^{2}$ is comparable to $-|\mu|^{2}$, and both are not larger than the square of a few hundred $\mathrm{GeV}$, so that the observed value of $m_{Z}$ could ensue without too much ${ }^{1}$

\footnotetext{
${ }^{1}$ Fine-tuning is an inherently fuzzy criterion. Therefore, I make no further attempt to quantify it, as it is not possible to do so in a purely scientific way. Nevertheless, it is certainly useful, and even necessary, for scientists as a personal and subjective guide for deciding how to allocate scarce resources such as time and money. The practical meaning of the words "too much" is therefore left to the reader.
} 
fine-tuning. The most prominent feature of the so-called natural supersymmetry scenario is that, since $|\mu|$ should not be too large, the Higgsinos should be relatively light.

Of course, the idea that natural supersymmetry requires small $|\mu|$ should be examined critically. Some proposals that seek to decouple the Higgsino masses from the supersymmetric little hierarchy problem in various ways have appeared in Refs. [2-5]. The present paper is motivated by the possibility that the little hierarchy problem can be ameliorated in another way that does not require small $|\mu|$, by finding a reason why the particular combination

$$
m_{H_{u}}^{2}+|\mu|^{2},
$$

which appears as the non-loop-suppressed part of Eq. (1.1), can be dynamically driven toward small values, even if the individual terms in it are not and even if all superpartner squared masses are much larger.

One possible approach to realization of this comes from conformal sequestering [6-13], the proposal that supersymmetry-breaking effects in the visible (MSSM) sector are renormalized by strong and nearly conformal dynamics in a hidden sector related to supersymmetry breaking. In a refinement of this idea, called scalar sequestering [14-16], the scalar squared masses have an extra suppression, compared to the dimension-1 parameters, due to the strong dynamics. This has the virtue of also ameliorating flavor violation problems that can arise in supersymmetry due to sfermion mixing. Other works that build on these ideas can be found in Refs. [17-23], and the idea that conformal dynamics coupled directly to the MSSM sector can suppress flavor violation has been proposed in Refs. [24,25]. From the point of view of the present paper, it is particularly intriguing that in theories of scalar sequestering, as pointed out in Refs. $[15,16]$, the dimension-2 parameters that undergo conformal scaling include the combined quantity $m_{H_{u}}^{2}+|\mu|^{2}$ (and not the individual parameters $m_{H_{u}}^{2}$ or $|\mu|^{2}$ ), just as called for in the preceding paragraph.

In this paper, I will reexamine this possibility, paying particular attention to the previously neglected fact that there is an interplay between the hidden sector and the visible sector contributions to the renormalization group (RG) running, which can lead to quasifixed point relations at intermediate scales. Here, it is particularly important to take into account restrictions [26-28] on the scaling of operators in superconformal field theories, which follow from unitarity and crossing symmetry and which constrain the extent to which $m_{H_{u}}^{2}+|\mu|^{2}$ can run. The emphasis is on the little hierarchy problem that has been exacerbated during the years of LHC searches and by the measurement of the Higgs boson mass at $125 \mathrm{GeV}$.

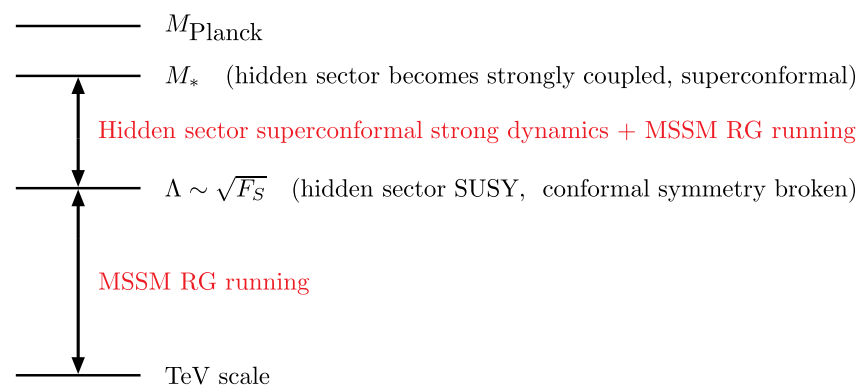

FIG. 1. The assumed hierarchies of scales involved in the communication of supersymmetry breaking from the hidden sector (where supersymmetry is spontaneously broken) to the visible sector (which contains the MSSM particles).

\section{REVIEW OF SCALAR SEQUESTERING}

Suppose that spontaneous supersymmetry breaking occurs in a strongly coupled hidden sector and is communicated to the MSSM sector by a singlet chiral superfield $S$ which has a nonzero $F$-term vacuum expectation value denoted $F_{S}$. The dynamics of the hidden sector, including $S$, enters into a superconformal scaling regime at a high scale $M_{*}$. The superconformal symmetry is then spontaneously broken at a lower scale to be denoted $\Lambda$, which is of order $\sqrt{F_{S}}$. The assumed hierarchies of scales are illustrated schematically in Fig. 1. The mass scale $M_{*}$ is ideally supposed to be much larger than $\Lambda$ (although this hierarchy is bounded, as pointed out in Ref. [23] and discussed below).

The leading nonrenormalizable terms that communicate supersymmetry breaking to the MSSM sector are

$$
\begin{array}{r}
\mathcal{L}_{\text {gaugino masses }}=-\frac{c_{a}}{2 M_{*}} \int d^{2} \theta S \mathcal{W}^{a \alpha} \mathcal{W}_{\alpha}^{a}+\text { c.c. } \\
\mathcal{L}_{a \text { terms }}=-\frac{c^{i j k}}{6 M_{*}} \int d^{2} \theta S \phi_{i} \phi_{j} \phi_{k}+\text { c.c. } \\
\mathcal{L}_{\mu \text { term }}=\frac{c_{\mu}}{M_{*}} \int d^{4} \theta S^{*} H_{u} H_{d}+\text { c.c. } \\
\mathcal{L}_{b \text { term }}=-\frac{c_{b}}{M_{*}^{2}} Z_{S^{*} S} \int d^{4} \theta S^{*} S H_{u} H_{d}+\text { c.c. } \\
\mathcal{L}_{m^{2} \text { terms }}=-\frac{c_{i}^{j}}{M_{*}^{2}} Z_{S^{*} S} \int d^{4} \theta S^{*} S \phi^{* i} \phi_{j},
\end{array}
$$

where the coefficients $c$ are dimensionless parameters. Note that there is another class of terms that could be written in the Lagrangian,

$$
-\frac{k_{i}^{j}}{M_{*}} \int d^{4} \theta S \phi^{* i} \phi_{j}+\text { c.c., }
$$


but these are redundant, as they can be eliminated in favor of the terms in Eqs. (2.4) and (2.5) and higher-order terms, by a holomorphic field redefinition $\phi_{i} \rightarrow \phi_{i}+k_{i}^{j} S \phi_{j} / M_{*}$.

The terms in Eqs. (2.1)-(2.5) are written in the holomorphic basis for $S$. In this basis, there is no hidden-sector renormalization of the gaugino masses, $a$ terms, or the $\mu$ term, because each of Eqs. (2.1)-(2.3) depends on either $S$ or $S^{*}$, but not both. In contrast, the $b$ term and the nonholomorphic scalar squared masses are not holomorphic in $S$ and so are renormalized by an extra suppression factor,

$$
Z_{S^{*} S}=\left(Q / Q_{0}\right)^{\Gamma}
$$

by the hidden-sector superconformal dynamics, where $Q$ is the renormalization scale, $Q_{0}$ is a reference scale, and

$$
\Gamma=\Delta_{S^{*} S}-2 \Delta_{S}
$$

in which

$$
\Delta_{S}=1+\gamma_{S},
$$

where $\gamma_{S}$ is the anomalous dimension of $S$ and $\Delta_{S^{*} S}$ is the lowest scaling dimension for a scalar operator appearing in the operator product expansion of $S^{*}$ and $S$. In this holomorphic basis, $S$ has a noncanonical kinetic term,

$$
\mathcal{L}_{\text {kinetic }}=Z_{S} \int d^{4} \theta S^{*} S,
$$

where the hidden-sector wave function renormalization factor is

$$
Z_{S}=\left(Q / Q_{0}\right)^{-2 \gamma_{S}}
$$

Now, one can go to a canonical basis for $S$, by making the redefinition

$$
S \rightarrow Z_{S}^{-1 / 2} S \text {. }
$$

In the canonical basis for $S$, the MSSM gaugino masses $\tilde{M}_{a}$, scalar cubic couplings $\tilde{a}^{i j k}, \tilde{\mu}$ term, holomorphic supersymmetry-breaking Higgs squared mass term $\tilde{b}$, and nonholomorphic supersymmetry-breaking scalar squared masses can be evaluated as ${ }^{2}$

$$
\begin{gathered}
\tilde{M}_{a}=c_{a} Z_{S}^{-1 / 2} F_{S} / M_{*}, \\
\tilde{a}^{i j k}=c^{i j k} Z_{S}^{-1 / 2} F_{S} / M_{*},
\end{gathered}
$$

\footnotetext{
${ }^{2}$ The reason for the tildes in the names of these dimensionful parameters is to distinguish them from their counterparts after a further redefinition to be made shortly.
}

$$
\begin{gathered}
\tilde{\mu}=c_{\mu} Z_{S}^{-1 / 2} F_{S}^{*} / M_{*}, \\
\tilde{b}=c_{b} Z_{S^{*} S} Z_{S}^{-1}\left|F_{S}\right|^{2} / M_{*}^{2}, \\
\left(\tilde{m}^{2}\right)_{i}^{j}=c_{i}^{j} Z_{S^{*} S} Z_{S}^{-1}\left|F_{S}\right|^{2} / M_{*}^{2} .
\end{gathered}
$$

The presence of the extra hidden-sector renormalization factor $Z_{S^{*} S}$ for the MSSM parameters of mass dimension 2 implies that they have a modified running for the range $\Lambda<Q<M_{*}$, throughout which the hidden sector is assumed to be nearly superconformal.

Now, if we use the generic notations $\tilde{M}_{A}$ and $\tilde{m}_{i}^{2}$ for parameters of mass dimensions 1 and 2, respectively, and take into account the visible sector renormalization in a canonical basis for the MSSM fields, then the renormalization group running for $Q>\Lambda$ is

$$
\frac{d}{d t} \tilde{M}_{A}=\gamma_{S} \tilde{M}_{A}+\beta_{\tilde{M}_{A}}^{\mathrm{MSSM}}
$$

$$
\frac{d}{d t} \tilde{m}_{i}^{2}=\left(\Gamma+2 \gamma_{S}\right) \tilde{m}_{i}^{2}+\beta_{\tilde{m}_{i}^{2}}^{\mathrm{MSSM}},
$$

where $\beta_{\tilde{M}_{A}}^{\mathrm{MSSM}}$ and $\beta_{\tilde{m}_{i}^{2}}^{\mathrm{MSSM}}$ are the usual beta functions obtained without including hidden-sector effects, and

$$
t \equiv \ln \left(Q / Q_{0}\right) .
$$

To simplify the renormalization group running in practice, it is convenient to make a redefinition to undo the effect of going to the canonical basis for $S$, but remain in the canonical basis for the MSSM fields, by now defining, for $Q \geq \Lambda$

$$
\begin{aligned}
& M_{A}=(\Lambda / Q)^{\gamma_{S}} \tilde{M}_{A}, \\
& m_{i}^{2}=(\Lambda / Q)^{2 \gamma_{S}} \tilde{m}_{i}^{2},
\end{aligned}
$$

which then run according to

$$
\begin{gathered}
\frac{d}{d t} M_{A}=\beta_{M_{A}}^{\mathrm{MSSM}}, \\
\frac{d}{d t} m_{i}^{2}=\Gamma m_{i}^{2}+\beta_{m_{i}^{2}}^{\mathrm{MSSM}},
\end{gathered}
$$

where it is easy to check using dimensional analysis that $\beta_{M_{A}}^{\mathrm{MSSM}}$ and $\beta_{m_{i}^{2}}^{\mathrm{MSSM}}$ are obtained from $\beta_{\tilde{M}_{A}}^{\mathrm{MSSM}}$ and $\beta_{\tilde{m}_{i}^{2}}^{\mathrm{MSSM}}$ by simply substituting $\tilde{M}_{A} \rightarrow M_{A}$ and $\tilde{m}_{i}^{2} \rightarrow m_{i}^{2}$. Note that $\gamma_{S}$ has thus been eliminated from the running. For simplicity, $\Gamma$ is taken here to be a positive constant for $Q>\Lambda$, corresponding to an idealized exactly superconformal theory in the hidden sector, while $\Gamma=0$ for $Q<\Lambda$, where the superconformal symmetry of the hidden sector is broken. At the scale $Q=\Lambda$, the parameters are assumed 
to simply match, although in a more complete realistic model, they are likely governed by more complicated threshold corrections, and $\Gamma$ will not be exactly constant.

A crucial subtlety is that the $\mu$-term hidden-sector renormalization feeds [14-16] into that of the nonholomorphic Higgs scalar squared masses, so that the combinations that are subject to the hidden-sector scaling are actually $[15,16]$ the full nonholomorphic scalar squared mass combinations,

$$
\begin{aligned}
& \hat{m}_{H_{u}}^{2} \equiv m_{H_{u}}^{2}+|\mu|^{2}, \\
& \hat{m}_{H_{d}}^{2} \equiv m_{H_{d}}^{2}+|\mu|^{2},
\end{aligned}
$$

rather than the supersymmetry-breaking parameters $m_{H_{u}}^{2}$ and $m_{H_{d}}^{2}$. Equations (2.23) and (2.24) therefore apply to the MSSM parameters:

$M_{A}=$ gaugino masses, $a$ terms, and the $\mu$ term,

$m_{i}^{2}=$ squark and slepton squared masses, $\hat{m}_{H_{u}}^{2}, \hat{m}_{H_{d}}^{2}$, and $b$.

In the remainder of this paper, I will stick to the scheme in which Eqs. (2.23) and (2.24) hold, with boundary conditions for the input parameters of Eqs. (2.27) and (2.28) to be specified at the scale $Q=M_{*}$. [At that scale, one has the equivalences $\tilde{M}_{A}=\left(M_{*} / \Lambda\right)^{\gamma_{S}} M_{A}$ and $\tilde{m}_{i}^{2}=\left(M_{*} / \Lambda\right)^{2 \gamma_{S}} m_{i}^{2}$, while at the matching scale, $Q=\Lambda, \tilde{M}_{A}=M_{A}$ and $\tilde{m}_{i}^{2}=m_{i}^{2}$.]

In the numerical results below, I will use the two-loop MSSM beta functions found in Refs. [29-32]. The $\hat{m}_{H_{u}}^{2}$ and $\hat{m}_{H_{d}}^{2}$ MSSM beta functions are obtained straightforwardly from these, for example,

$$
\begin{aligned}
\beta_{\hat{m}_{H_{u}}^{2}}^{\mathrm{MSSM}}= & \frac{1}{16 \pi^{2}}\left[6 y_{t}^{2}\left(\hat{m}_{H_{u}}^{2}+m_{Q_{3}}^{2}+m_{u_{3}}^{2}\right)+\left(6 y_{b}^{2}+2 y_{\tau}^{2}\right) \mu^{2}\right. \\
& \left.+6 a_{t}^{2}-6 g_{2}^{2}\left(M_{2}^{2}+\mu^{2}\right)-\frac{6}{5} g_{1}^{2}\left(M_{1}^{2}+\mu^{2}-T / 2\right)\right] \\
& +\cdots, \\
\beta_{\hat{m}_{H_{d}}^{2}}^{\mathrm{MSSM}}= & \frac{1}{16 \pi^{2}}\left[6 y_{b}^{2}\left(\hat{m}_{H_{d}}^{2}+m_{Q_{3}}^{2}+m_{d_{3}}^{2}\right)\right. \\
& +2 y_{\tau}^{2}\left(\hat{m}_{H_{d}}^{2}+m_{L_{3}}^{2}+m_{\bar{e}_{3}}^{2}\right)+6 y_{t}^{2} \mu^{2}+6 a_{b}^{2} \\
& +2 a_{\tau}^{2}-6 g_{2}^{2}\left(M_{2}^{2}+\mu^{2}\right) \\
& \left.-\frac{6}{5} g_{1}^{2}\left(M_{1}^{2}+\mu^{2}+T / 2\right)\right]+\cdots
\end{aligned}
$$

where the ellipses represent the contributions beyond one-loop order and $g_{1}$ and $g_{2}$ are the electroweak gauge couplings in a grand unified theory (GUT) normalization, and

$$
T=\hat{m}_{H_{u}}^{2}-\hat{m}_{H_{d}}^{2}+\sum_{i=1}^{3}\left[m_{Q_{i}}^{2}-m_{L_{i}}^{2}-2 m_{u_{i}}^{2}+m_{d_{i}}^{2}+m_{e_{i}}^{2}\right] .
$$

\section{QUASIFIXED POINTS FROM INTERPLAY OF HIDDEN AND VISIBLE RENORMALIZATION}

In earlier work, it has often been assumed that $\Gamma$ is large and positive, so that the MSSM contributions to the running of the dimension-2 parameters are relatively negligible for $Q>\Lambda$. In the idealized limit of large $\Gamma$, there is power-law running resulting in a relative suppression $\left(\Lambda / M_{*}\right)^{\Gamma}$ for the dimension-2 terms, compared to the squares of dimension- 1 terms, at the scale $Q=\Lambda$. In that limit, one naively can impose boundary conditions

$$
m_{i}^{2} \approx 0
$$

at $Q=\Lambda$, provided that there is a significant hierarchy $\Lambda / M_{*}$.

However, constraints on superconformal field theories have shown $[26,27]$ that, while $\Gamma$ indeed might be positive, it cannot be too large, with stronger bounds for smaller $\gamma_{S}$. These papers have also provided some circumstantial evidence for the existence of a minimal superconformal theory, which may (based on extrapolation of established constraints) have

$$
\begin{gathered}
\gamma_{S} \approx 3 / 7, \\
\Gamma \lesssim 0.3,
\end{gathered}
$$

although there is so far no specific identification of this theory or guarantee of its existence. The constraints found in Refs. [26-28] also imply that any significantly smaller $\gamma_{S}$ would necessarily have a much smaller $\Gamma$.

From these constraints, in any given model, there is a limit [23] on the range of scales at which the hidden sector can remain in the superconformal regime, based on existing LHC bounds on the superpartner masses. Taking $\Lambda=\sqrt{F_{S}}$ and $c_{3}$ of order unity, and requiring that at the scale $Q=\Lambda$ the gluino mass $M_{3} \sim c_{3}\left(F_{S} / M_{*}\right)\left(\Lambda / M_{*}\right)^{\gamma_{S}}$ exceeds $1000 \mathrm{GeV}$, this constraint amounts to roughly

$$
\Lambda \gtrsim\left[(1000 \mathrm{GeV}) M_{*}^{1+\gamma_{S}}\right]^{1 /\left(2+\gamma_{S}\right)} .
$$

Now, taking $\gamma_{S} \gtrsim 3 / 7$ and identifying $M_{*}$ with the scale $M_{\mathrm{GUT}}=2.5 \times 10^{16} \mathrm{GeV}$ at which the gauge couplings appear to unify, one finds that $\Lambda \gtrsim 8 \times 10^{10} \mathrm{GeV}$. 
In the following, I will therefore optimistically take $M_{*}=M_{\mathrm{GUT}}$ and $\Lambda=10^{11} \mathrm{GeV}$ and $\Gamma=0.3$ for numerical examples. ${ }^{3}$ For this, or smaller, values of $\Gamma$, a more accurate treatment than Eq. (3.1) is that the dimension-2 MSSM parameters are drawn toward a quasifixed point trajectory solution at which the MSSM contributions to the beta functions balance with the hidden-sector contributions, so that the right side of Eq. (2.24) approximately vanishes. The quasifixed point trajectories for the dimension-2 parameters therefore can be roughly approximated as the solutions of the algebraic equations

$$
m_{i, \text { quasifixed }}^{2} \approx-\beta_{m_{i}^{2}}^{\mathrm{MSSM}} / \Gamma,
$$

where the dimension-2 parameters appearing in the MSSM beta functions on the right-hand side are self-consistently set equal to their quasifixed point values. However, note that these quasifixed points are moving targets, which in practice means that the right-hand side of Eq. (3.5) should be evaluated at a scale slightly larger than the left-hand side. Equation (3.5) does provide useful approximate relations discussed in the next few paragraphs, but rather than attempting a more detailed and precise analytical expression for the quasifixed point trajectories, in the examples of the next section, the running will be evaluated numerically.

Fortunately, the MSSM beta functions for squark and slepton squared masses come mostly from gaugino masses, and are negative. This means that the squared masses will tend to approach positive quasifixed point values at the matching scale $Q=\Lambda$. For example, the rough estimate for the quasifixed point of the running right-handed selectron mass, in terms of the running bino mass parameter $M_{1}$ and the $U(1)_{Y}$ gauge coupling in a GUT normalization $g_{1}$, is:

$m_{\tilde{e}_{R}, \text { quasifixed }} \approx \sqrt{\frac{3}{10}} \frac{g_{1} M_{1}}{\pi \sqrt{\Gamma}}=0.18\left(\frac{g_{1}}{0.57}\right)\left(\frac{0.3}{\Gamma}\right)^{1 / 2} M_{1}$,

rather than 0 , and for a typical squark mass, including only the effects of the running gluino mass $M_{3}$ :

$m_{\tilde{q}, \text { quasifixed }} \approx \sqrt{\frac{2}{3}} \frac{g_{3} M_{3}}{\pi \sqrt{\Gamma}}=0.365\left(\frac{g_{3}}{0.77}\right)\left(\frac{0.3}{\Gamma}\right)^{1 / 2} M_{3}$.

These expressions have been normalized to typical values for the MSSM running gauge couplings at an intermediate scale $\Lambda=10^{11} \mathrm{GeV}$.

\footnotetext{
${ }^{3}$ Anomaly-mediation [33] contributions of order $F_{S} / 16 \pi^{2}$ $M_{\text {Planck }}$ to gaugino masses and $F_{S}^{2} /\left(16 \pi^{2} M_{\text {Planck }}\right)^{2}$ to scalar squared masses are neglected here but could be significant for larger choices of $M_{*}$.
}

However, as noted above, the quasifixed points are moving targets, because the gaugino masses (and other contributions to the MSSM beta functions) are running with the scale $Q$. Another important practical effect is that in realistic models the quasifixed point trajectories are not actually reached with the finite running available from $M_{*}$ down to $\Lambda$, so the above estimates are not quite realized. As we will see, at $Q=\Lambda$ the running masses of the squarks and especially the sleptons are often considerably higher than the estimates of eqs. (3.6) and (3.7) would indicate. There is also a significant effect due to the subsequent running from the scale $\Lambda$ down to the TeV scale. The effect of running below $\Lambda$ is actually the dominant effect for the physical squark masses (because $M_{3}$ and $g_{3}$ are growing in the infrared), but it is relatively much smaller for sleptons (because $M_{1}$ and $g_{1}$ are shrinking in the infrared). Therefore, the influence of the quasifixed point behavior given by eq. (3.5) turns out to be crucial for understanding how sleptons can be heavy enough to avoid discovery at the LHC, and also how the lightest supersymmetric particle (LSP) need not be a charged slepton.

The quasifixed point behavior of the Higgs squared masses is of even greater importance. From Eqs. (2.29) and (3.5), one obtains an estimate for the running quasifixed point trajectory, for $Q$ much less than $M_{*}$ but not smaller than $\Lambda$ :

$$
\begin{aligned}
& \hat{m}_{H_{u}, \text { quasifixed }}^{2} \\
& \approx \frac{3}{8 \pi^{2} \Gamma+3 y_{t}^{2}}\left[g_{2}^{2}\left(M_{2}^{2}+\mu^{2}\right)+\frac{g_{1}^{2}}{5}\left(M_{1}^{2}+\mu^{2}-T / 2\right)-a_{t}^{2}\right. \\
& \left.-y_{t}^{2}\left(m_{Q_{3}}^{2}+m_{u_{3}}^{2}\right)-\mu^{2}\left(y_{b}^{2}+y_{\tau}^{2} / 3\right)\right] \text {. }
\end{aligned}
$$

Realistic electroweak symmetry breaking requires that $\hat{m}_{H_{u}}^{2}$ must be small in magnitude near the $\mathrm{TeV}$ scale; this is the essence of the supersymmetric little hierarchy problem. Because Eq. (3.8) has significant contributions of both signs, and is suppressed by $8 \pi^{2} \Gamma$, one can adjust it to the appropriate value, even if $a_{t}, \mu, M_{2}, M_{1}, m_{Q_{3}}$, and $m_{u_{3}}$ are much larger in magnitude than a TeV. As a guide to finding models with correct electroweak symmetry breaking, note that to decrease the low-energy prediction for $\hat{m}_{H_{u}}^{2}$, one can increase $\left|a_{t}\right|, m_{Q_{3}}^{2}$, or $m_{u_{3}}^{2}$ or decrease $|\mu|,\left|M_{2}\right|$, or $\left|M_{1}\right|$. However, the subsequent running from $Q=\Lambda$ down to the $\mathrm{TeV}$ scale is also quite significant, so that the actual value of $\hat{m}_{H_{u}}^{2}$ that is needed at $Q=\Lambda$ is not an extremely small value. The requirement of correct electroweak symmetry breaking can easily be obtained by adjusting the input parameters in a predictive way, but the level of tuning required, while arguably reduced as illustrated in examples below, cannot be said to be eliminated.

The other dimensionful quantities appearing in the Higgs potential, $\hat{m}_{H_{d}}^{2}$ and $b$, are also strongly influenced to flow toward quasifixed point trajectories in the infrared. 
This implies that to some approximation, the information about the initial conditions at $Q=M_{*}$ is washed out, and roughly speaking, the Higgs potential parameters are predicted in terms of the dimension-1 parameters of the theory. Despite the fact that this washing out is incomplete due to the quasifixed points not quite being reached, there are some robust trends that remain in the form of constraints and correlations between the soft supersymmetrybreaking parameters of the theory at the TeV scale. These will be explored numerically for some sample slices in parameter space in the next section.

\section{NUMERICAL EXAMPLES}

In this section, I provide some examples that illustrate the importance of the quasifixed point behavior in the presence of scalar sequestering dynamics. The low-energy results are only weakly dependent on the high-scale boundary condition values of the dimension-2 parameters. Therefore, for simplicity and to keep the dimensionality of parameter space small, I will take all of the squark and slepton squared masses and $\hat{m}_{H_{u}}^{2}$ and $\hat{m}_{H_{d}}^{2}$ to be equal to a common value $m_{0}^{2}$ at $Q=M_{*}$. Also for simplicity, ${ }^{4} \mathrm{I}$ assume that the scalar trilinear couplings are governed by a universality condition, with $a_{t}=A_{0} y_{t}$ and $a_{b}=A_{0} y_{b}$ and $a_{\tau}=A_{0} y_{\tau}$ at $Q=M_{*}$; with this assumption, the particular values of $a_{b}$ and $a_{\tau}$ are of much less importance than that of $a_{t}$. The dimensionful input parameters are therefore

$$
M_{1}, M_{2}, M_{3}, A_{0}, \mu, m_{0}^{2}, b,
$$

specified at the renormalization scale $Q=M_{*}$. The RG scale at which superconformal running begins in the hidden sector is $M_{*}=M_{\mathrm{GUT}}=2.5 \times 10^{16} \mathrm{GeV}$, and the superconformal running regime ends at $\mathrm{RG}$ scale $\Lambda=10^{11} \mathrm{GeV}$. The parameters of the theory are run using Eqs. (2.23) and (2.24) with $\Gamma=0.3$ from $Q=M_{*}$ down to $Q=\Lambda$ and then with $\Gamma=0$ from $Q=\Lambda$ to the electroweak scale.

As a constraint on the parameter space, the requirement of correct electroweak symmetry breaking with $m_{Z}=$ $91 \mathrm{GeV}$ and a fixed value of $\tan \beta$ are imposed. This allows for two parameters to be solved for, which I take to be $A_{0}$ and $\mu$. (It is better to avoid choosing $m_{0}^{2}$ or $b$ as a parameter to be solved for, due to the much weaker dependence of the Higgs potential on their high-scale values, which follows from the quasifixed point behavior.) In practice, the parameters $A_{0}$ and $\mu$ are solved for by iterating to convergence, starting from an arbitrary initial

\footnotetext{
${ }^{4}$ This is a quite nontrivial assumption from the point of view of the supersymmetric flavor problem, since completely general scalar cubic interactions could be dangerous. However, imposing a flavor symmetry on these terms is technically natural, and the contributions to scalar squared masses mediated by RG running from the gaugino masses are flavor blind and could dominate the sfermion mixings.
}

guess. The lightest Higgs boson mass $M_{h}$ is obtained using the leading three-loop calculation given in Ref. [34], augmented by one-loop electroweak corrections. In recognition of the theoretical and parametric uncertainties [35] in the $M_{h}$ calculation, this predicted value is required to be in the range $123 \mathrm{GeV}<M_{h}<127 \mathrm{GeV}$. The $M_{h}$ constraint has the greatest impact on the allowed values of $M_{3}$ (which feeds into the magnitudes of the top-squark masses) and $A_{0}$ (which controls the top-squark mixing). Search limits on direct superpartner production at the LHC turn out to not constrain the models given below, because the Higgs mass constraint indirectly requires the gluino and squarks to be heavy anyway.

\section{A. Pessimistic case: Unified gaugino mass boundary conditions}

In this subsection, I consider models that have unified gaugino mass parameters $M_{1}=M_{2}=M_{3}=m_{1 / 2}$ at $Q=$ $M_{*}$ and $\tan \beta=15$ fixed. The electroweak symmetrybreaking constraint then turns out to require that $A_{0}$ is positive (see Fig. 5 below), which in turn implies that at the electroweak scale $a_{t}$ is negative but not very large in magnitude, so that top-squark mixing is moderate. Because of this, to obtain $M_{h}$ in agreement with experiment requires a rather large $m_{1 / 2}$, between about 2.7 and $8.3 \mathrm{TeV}$ to obtain an estimated $123 \mathrm{GeV}<M_{h}<127 \mathrm{GeV}$.

The quasifixed point behavior of the RG running with $Q$ is shown for the choice $m_{1 / 2}=4500 \mathrm{GeV}$, for various boundary condition values of the remaining independent input parameter $m_{0}^{2}=b$, in Figs. 2 and 3. The trajectories for $\hat{m}_{H_{u}}^{2}$ converge to quasifixed point values near $(2 \mathrm{TeV})^{2}$ in this model, in good agreement with Eq. (3.8). The fact that this is not much smaller illustrates the importance of including the visible sector renormalization effects for $Q>\Lambda$ together with the hidden-sector scaling. Even for very large $m_{0}^{2}$, shown up to $(10 \mathrm{TeV})^{2}$ in the figure, the value of $\hat{m}_{H_{u}}^{2}$ at $Q=\Lambda$ is only slightly higher. The running of $\hat{m}_{H_{u}}^{2}$ down to the $\mathrm{TeV}$ scale is quite substantial, but Fig. 2(a) shows that there is an additional focusing effect for $Q<\Lambda$ that helps to make the low-energy trajectory rather insensitive to the high-scale value of $m_{0}$. However, the model cannot be viewed as free of fine-tuning, because the values of $A_{0}$ and $\mu$ have to be chosen rather precisely in this case to ensure that $\hat{m}_{H_{u}}^{2}$ runs close to 0 at the appropriate $\mathrm{RG}$ scale of a few $\mathrm{TeV}$ in order to obtain $m_{Z}=91 \mathrm{GeV}$. This can be appreciated by noting the large magnitude of the slope of the running of $\hat{m}_{H_{u}}^{2}$ for $Q$ below the $10 \mathrm{TeV}$ scale.

The other two panels of Fig. 2 show the quasifixed point behavior for the other two dimensionful Lagrangian parameters appearing in the tree-level Higgs potential, $\hat{m}_{H_{d}}^{2}$ and $B=b / \mu$. Note that $\hat{m}_{H_{d}}^{2}$ also flows to values of order $(2 \mathrm{TeV})^{2}$ at the intermediate scale but is not as strongly modified by the running below this. The running 

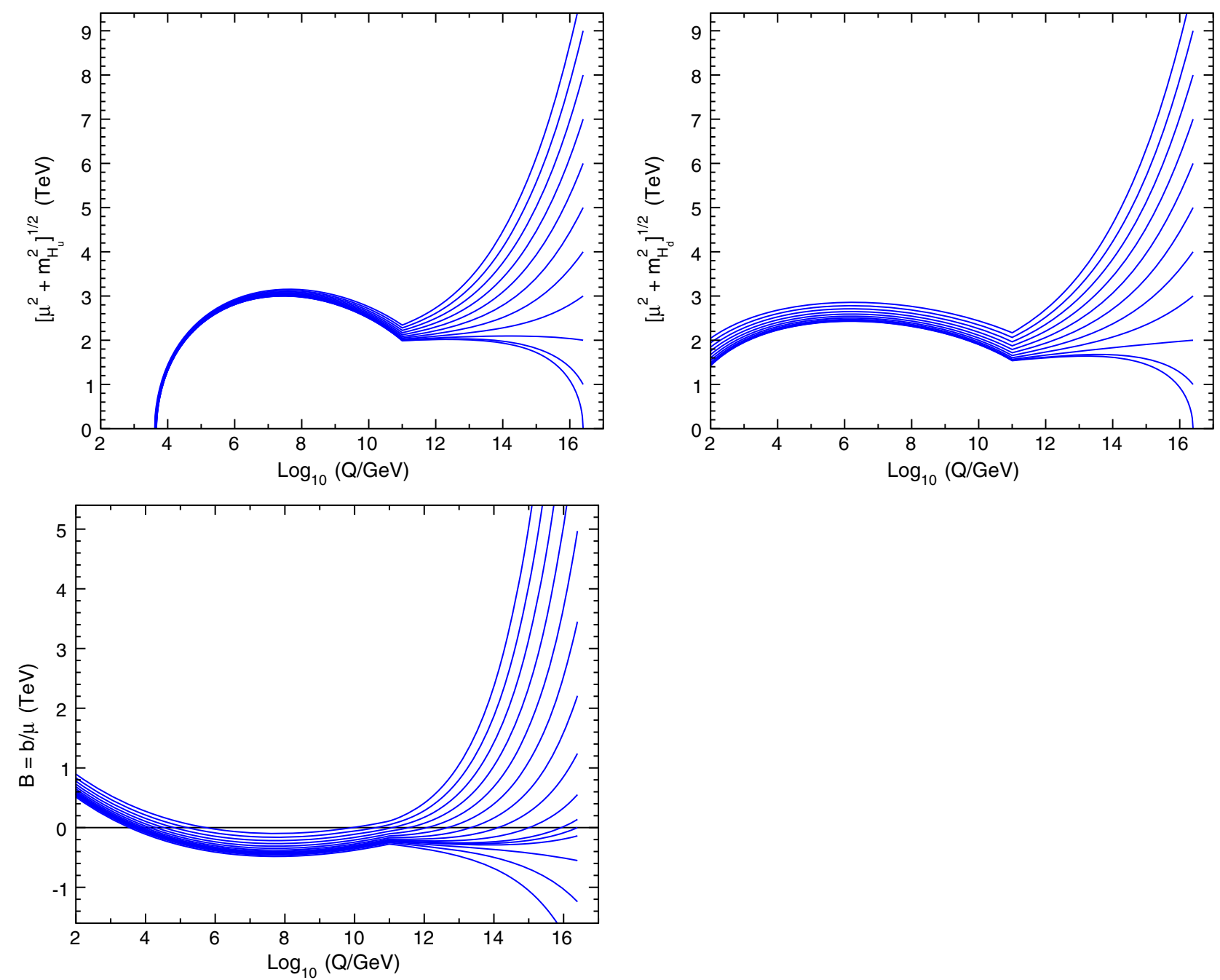

FIG. 2. Renormalization group running of the Higgs mass parameters $\left(m_{H_{u}}^{2}+\mu^{2}\right)^{1 / 2},\left(m_{H_{d}}^{2}+\mu^{2}\right)^{1 / 2}$, and $B=b / \mu$, as a function of the RG scale $Q$, for a model with $M_{1}=M_{2}=M_{3}=4500 \mathrm{GeV}$ at $M_{\mathrm{GUT}}$, and $\Gamma=0.3$ and $\Lambda=10^{11} \mathrm{GeV}$. Each line is the RG trajectory for a different boundary condition of the common scalar squared mass $m_{0}^{2}=b$ at the GUT scale, showing the approach to the quasifixed point behavior at low RG scales.

of $B$ flows toward small negative values at the intermediate scale, and it runs positive near the $\mathrm{TeV}$ scale but does not exceed a few hundred $\mathrm{GeV}$ in magnitude. In both of these cases, the focusing (both from the quasifixed point nature of the running above $\Lambda$ and the subsequent running below $\Lambda)$ is not as pronounced as for $\hat{m}_{H_{u}}^{2}$.

The first panel of Fig. 3 shows the quasifixed point influence on the running of a typical squark mass, in this case $\tilde{u}_{R}$. For comparison, the running gluino mass parameter $M_{3}$, which mostly drives the squark masses, is also shown. Again, the quasifixed point value of the squark masses at $Q=\Lambda$ is of order $2 \mathrm{TeV}$ [somewhat larger than the rough prediction of Eq. (3.7)], but the effect of pure MSSM running for $Q<\Lambda$ dominates over this, resulting in squark masses at the weak scale that are of order $0.8 M_{3}$, even if the initial value $m_{0}$ is much larger or smaller. This is a robust prediction of the framework, and it depends only weakly on $\Gamma$ or $\Lambda$, provided only that the latter is not too small. This is qualitatively similar to models with no-scale [36-38] or gaugino-mediated [39-41] boundary conditions.

The running of the right-handed selectron mass is shown in the right panel of Fig. 3. Here, it is apparent that the approach to the quasifixed point trajectory is much slower, resulting in a much larger spread of possible values for $m_{\tilde{e}_{R}}$ at $\Lambda$ [which generally exceed the rough prediction of Eq. (3.6)] and at the $\mathrm{TeV}$ scale. For comparison, also shown is the bino mass parameter $M_{1}$, which is mainly responsible for driving it. There is a competition for the role of the LSP between the lightest charged slepton and a binolike neutralino. If $m_{0} \lesssim 2.5 m_{1 / 2}$, then the LSP will be a charged slepton. To avoid cosmological problems from a charged stable LSP, one can invoke $R$-parity violation to allow the LSP to decay. Conversely, if $m_{0} \gtrsim 2.5 m_{1 / 2}$ at 

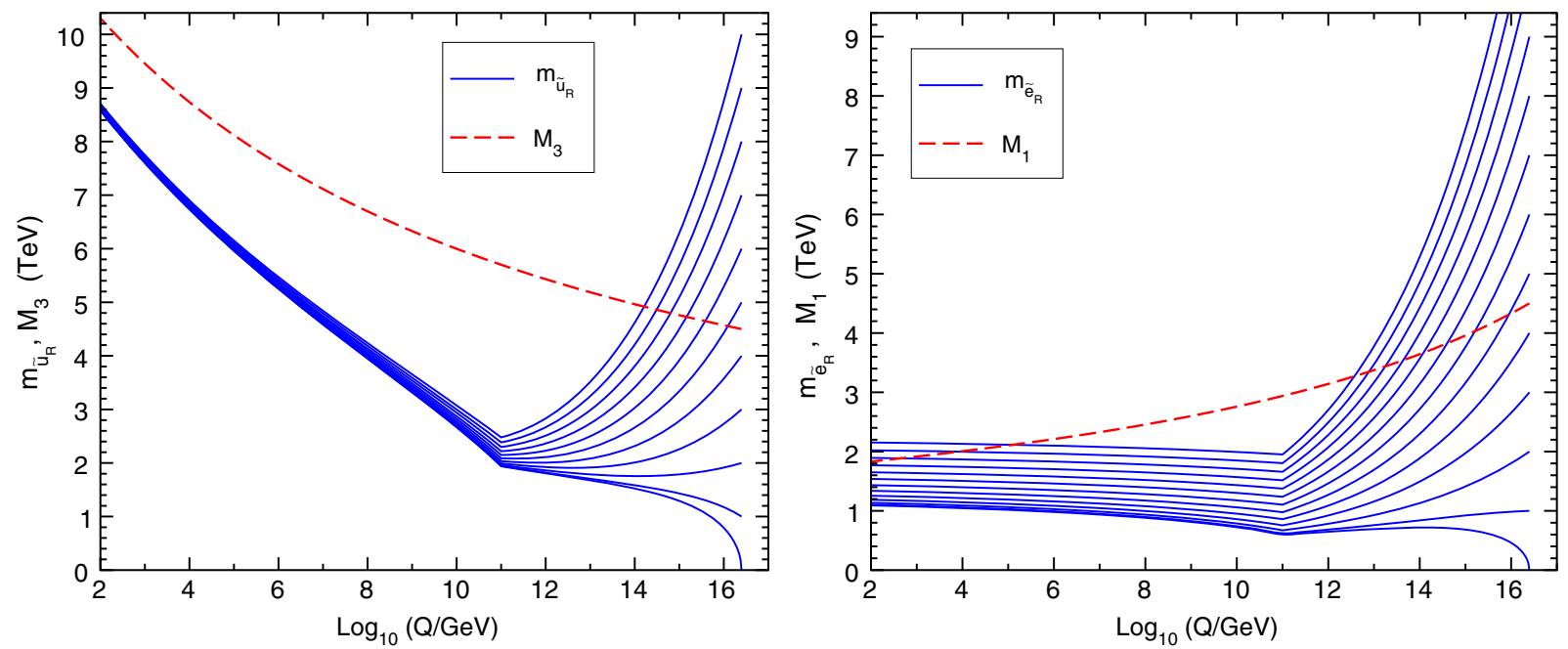

FIG. 3. RG running of the squark and slepton mass parameters $m_{\tilde{u}_{R}}$ (left panel) and $m_{\tilde{e}_{R}}$ (right panel) for a model with $M_{1}=M_{2}=M_{3}=4500 \mathrm{GeV}$ at $M_{\mathrm{GUT}}$, and $\Gamma=0.3$ and $\Lambda=10^{11} \mathrm{GeV}$. Each line is the RG trajectory for a different boundary condition of the common scalar mass at the GUT scale, showing the approach to the quasifixed point behavior. The running gluino and bino masses $M_{3}$ and $M_{1}$ are also shown for comparison.

$Q=M_{*}$, then the LSP will be a neutralino and could in principle be the dark matter, if $R$-parity is conserved. Obtaining a correct thermal relic abundance from the early Universe may require some fine adjustment of the masses, to enable the stau coannihilation mechanism for example. The dividing line between these two cases is rather robust and generally given by $m_{\tilde{e}_{R}} \approx 2.5 M_{1}$ at $Q=M_{*}$ even in models without gaugino mass unification, because to a good approximation, only $M_{1}$ enters into the quasifixed point attraction and subsequent running below $\Lambda$ for the right-handed slepton masses.

Figure 4 shows the spectrum of the physical masses of selected superpartners and the heavier Higgs bosons as a function of the universal gaugino mass $m_{1 / 2}$ at $Q=M_{*}$, for the two choices $m_{0}=m_{1 / 2}$ and $m_{0}=2.5 m_{1 / 2}$, with $b=m_{1 / 2}^{2}$ in both cases. The range of $m_{1 / 2}$ on the horizontal
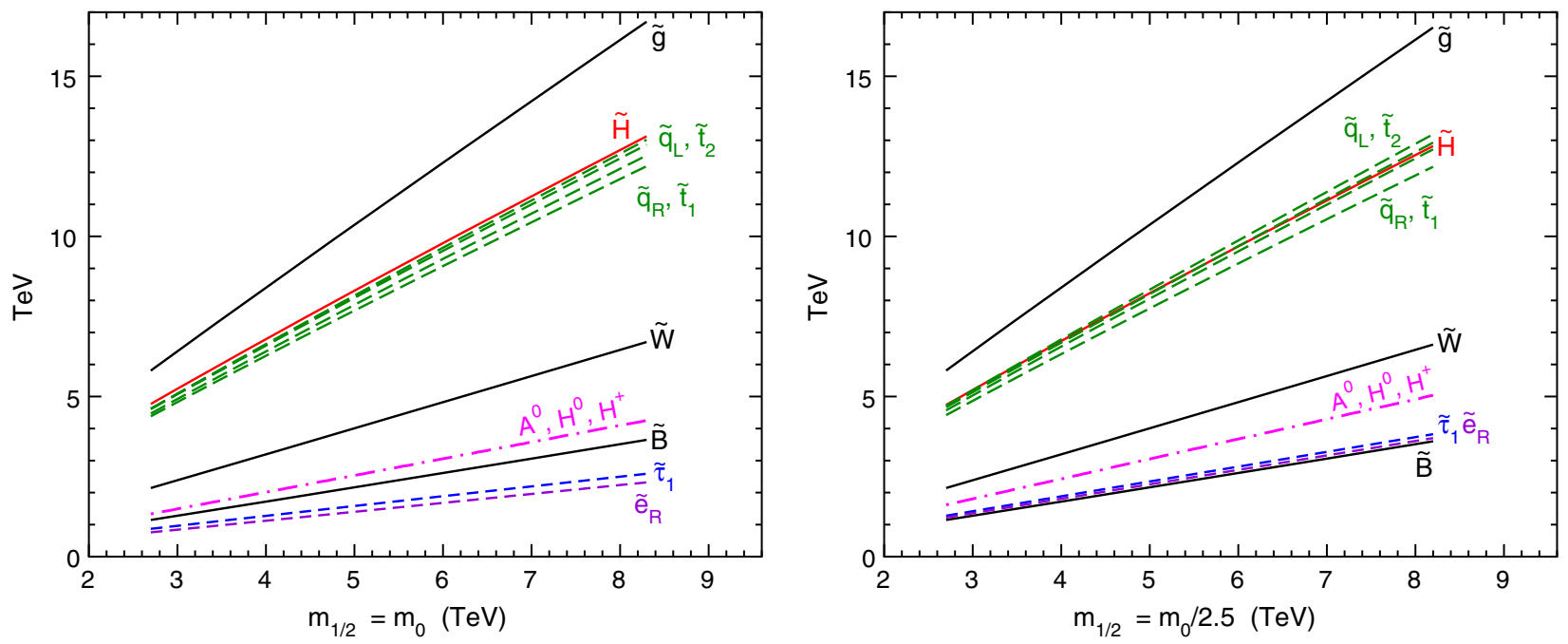

FIG. 4. The spectrum of physical masses of the gluino, squarks, sleptons, electroweakinos, and the heavier Higgs bosons, for a model line with varying GUT-scale input parameter $m_{1 / 2}$, with calculated $M_{h}$ between 123 (lower edge of $m_{1 / 2}$ ) and $127 \mathrm{GeV}$ (higher edge). The scalar trilinear coupling parameter $A_{0}$ and the Higgsino mass parameter $\mu$ are determined by requiring electroweak symmetry breaking with $m_{Z}=91 \mathrm{GeV}$ and $\tan \beta=15$. In the left panel, the common scalar mass is $m_{0}=m_{1 / 2}$, and the LSP is a slepton. In the right panel, $m_{0}=2.5 m_{1 / 2}$, and the LSP is a binolike neutralino. In both cases, $b=m_{1 / 2}^{2}$ is imposed at the GUT scale. The solid lines are, from top to bottom, the gluino, Higgsino, wino, and bino. The long-dashed lines are squarks, the short-dashed lines are sleptons, and the dot-dashed lines are the pseudoscalar Higgs boson $A^{0}$ and the nearly degenerate charged and heavy neutral Higgs scalar bosons $H^{0}, H^{ \pm}$. 

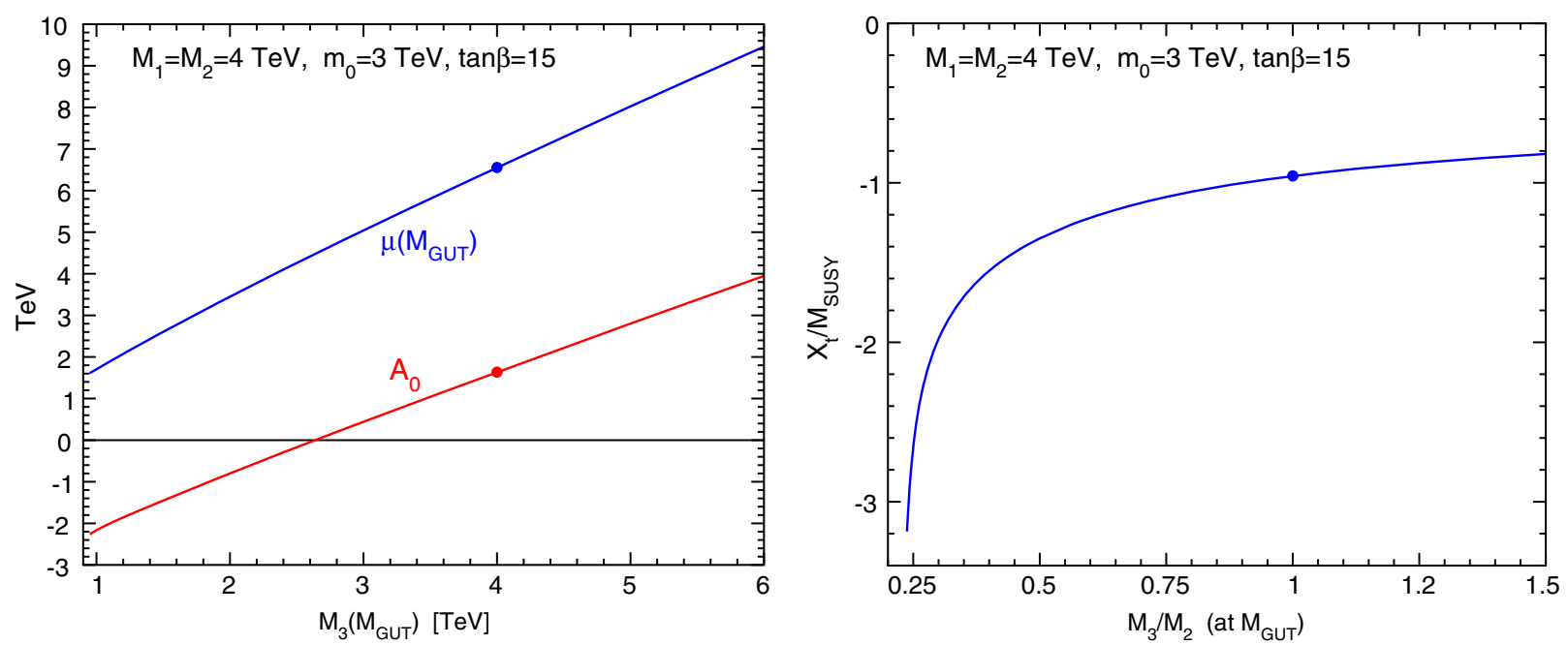

FIG. 5. Results obtained by solving the electroweak symmetry-breaking conditions $m_{Z}=91 \mathrm{GeV}$ and tan $\beta=15$, for models with fixed GUT-scale parameters $M_{1}=M_{2}=4 \mathrm{TeV}, m_{0}=3 \mathrm{TeV}$, and $b=(2 \mathrm{TeV})^{2}$, as a function of varying $M_{3}$. The left panel shows the solutions for the GUT-scale parameters $A_{0}$ and $\mu\left(M_{\mathrm{GUT}}\right)$. The right panel shows the resulting top-squark mixing parameter $X_{t} / M_{\mathrm{SUSY}}$ at $Q=M_{\mathrm{SUSY}}$, where $X_{t}=a_{t} / y_{t}-\mu / \tan \beta$ and $M_{\mathrm{SUSY}}=\sqrt{m_{\tilde{t}_{1}} m_{\tilde{t}_{2}}}$. The dots are the special case of gaugino mass unification.

axis shown for this spectrum corresponds to the estimated $123 \mathrm{GeV}<M_{h}<127 \mathrm{GeV}$. The general features to be observed include the following:

(i) The gluino is the heaviest superpartner, with a mass of at least about $6 \mathrm{TeV}$.

(ii) The squarks are also too heavy to produce with significant rates at the LHC unless there is a major beam energy upgrade.

(iii) The $\mu$ term is predicted to be very large, resulting in very heavy Higgsino-like charginos and neutralinos also beyond the LHC reach.

(iv) The heavier Higgs scalar bosons $A^{0}, H^{0}$, and $H^{ \pm}$are nearly degenerate and have masses well in excess of a TeV.

(v) The lightest stau is slightly heavier than the righthanded selectron and smuon, which escape being the LSP only if $m_{0} \gtrsim 2.5 m_{1 / 2}$, as noted above.

\section{B. Optimistic case: Nonunified gaugino mass boundary conditions}

The model line with unified gaugino masses considered above paints a rather bleak picture for the prospects of discovering anything new at the LHC. The reason for this is that the model predicts top-squark mixing that is not very large, so that accommodating $M_{h}$ near $125 \mathrm{GeV}$ requires large $M_{3}$, because this is the dominant parametric source for the necessary large top-squark masses.

A much lighter superpartner mass spectrum, with hopes for LHC discovery, can be achieved if one instead considers nonuniversal gaugino masses. By taking $M_{3}<M_{2}$ at the input scale, the quasifixed point trajectories given approximately by Eq. (3.8), and similar results for $\hat{m}_{H_{d}}^{2}$ and $b$, provide for correct electroweak symmetry breaking with $a_{t}$ negative and larger in magnitude compared to $M_{3}$. This in turn provides for large top-squark mixing, so that $M_{h}$ can be close to $125 \mathrm{GeV}$ consistent with relatively much lighter squarks (and the gluino) than found in the previous section with gaugino mass unification.

To illustrate this, consider the solutions for $A_{0}$ and $\mu$ obtained by varying $M_{3}$ while keeping fixed the input values $M_{1}=M_{2}=4 \mathrm{TeV}$ and all nonholomorphic scalar squared masses fixed at $m_{0}=3 \mathrm{TeV}$, with $b=(2 \mathrm{TeV})^{2}$ and $\tan \beta=15$. These are shown in the left panel of Fig. 5. The trend is for both $\mu$ and $A_{0}$ to decrease as $M_{3}$ is reduced. This implies that top-squark mixing is stronger for smaller $M_{3} / M_{2}$. In the right panel of Fig. 5, the ratio $X_{t} / M_{\text {SUSY }}$ at $Q=M_{\mathrm{SUSY}}$ is shown as a function of $M_{3} / M_{2}$ at $Q=M_{*}$. Here, $X_{t}=a_{t} / y_{t}-\mu / \tan \beta$ is a top-squark mixing parameter, and $M_{\text {SUSY }}$ is the geometric mean of the top-squark masses. It is well known that $\left|X_{t}\right| / M_{\text {SUSY }} \sim \sqrt{6}$ tends to approximately maximize $M_{h}$ for a given $M_{\text {SUSY }}$. As can therefore be inferred from Fig. 5(b), larger $M_{h}$ will ensue for $M_{3} / M_{2}<1$, with a particularly interesting range being about 0.25 to 0.5 for this ratio. ${ }^{5}$ The result is that for a fixed $M_{2}$ and varying $M_{3}$, the prediction for $M_{h}$ tends, somewhat coincidentally, to be surprisingly not too sensitive to $M_{3}$. This can be understood as due to larger $M_{3}$ providing for

\footnotetext{
${ }^{5}$ Nonuniversal gaugino masses, obtained for example if the $F$ term that breaks supersymmetry is a singlet under the Standard Model gauge group but transforms nontrivially under the GUT group as in Refs. [42-47], have also been used to address the supersymmetric little hierarchy problem. Coincidentally, naturalness in such models also prefers $M_{3} / M_{2} \sim 0.3$ at the GUT scale (see for example Refs. [48-59]), but for a quite different reason, as they work by making $m_{H_{u}}^{2}$ and $|\mu|^{2}$ individually small. These models are continuously connected [54] in parameter space to the focus-point scenario [60].
} 

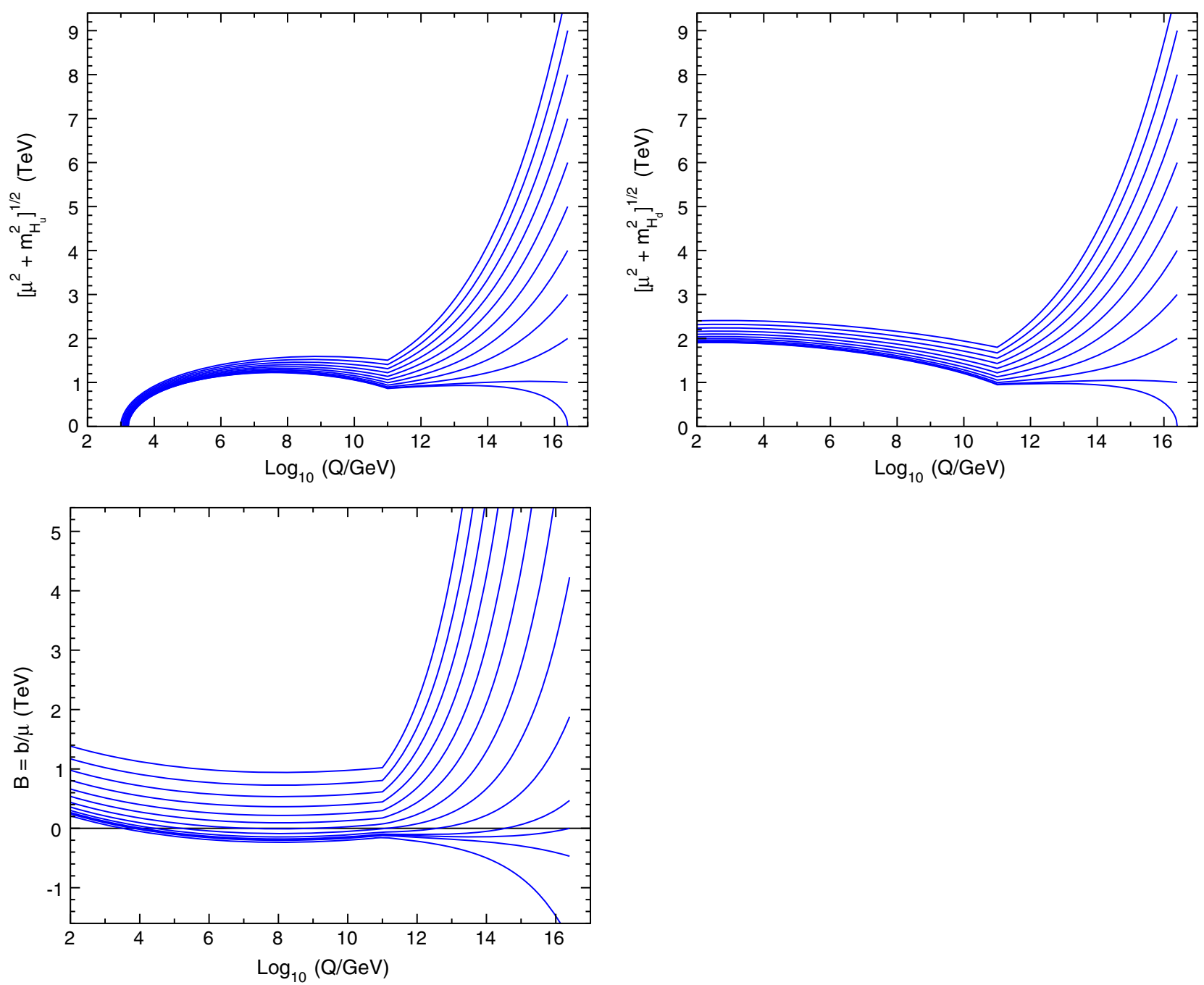

FIG. 6. Renormalization group running of the Higgs mass parameters $\left(m_{H_{u}}^{2}+\mu^{2}\right)^{1 / 2},\left(m_{H_{d}}^{2}+\mu^{2}\right)^{1 / 2}$, and $B=b / \mu$, for a model with nonuniversal gaugino masses $M_{1}=2000 \mathrm{GeV}, M_{2}=4000 \mathrm{GeV}$, and $M_{3}=1200 \mathrm{GeV}$ at the GUT scale. Each line is the RG trajectory for a different boundary condition of the common scalar squared mass $m_{0}^{2}=b$ at the GUT scale, showing the approach to the quasifixed point behavior at low RG scales.

larger logarithmic contributions to $M_{h}$ from the overall magnitude of the top-squark mass scale, while smaller $M_{3}$ yields larger top-squark mixing contributions to $M_{h}$.

Consider an example model line defined by gaugino masses chosen at $M_{*}$ to be $M_{3}=1200 \mathrm{GeV}, M_{2}=$ $4000 \mathrm{GeV}$, and $M_{1}=2000 \mathrm{GeV}$, and varying universal $m_{0}^{2}=b$, with $A_{0}$ and $\mu$ determined by correct electroweak symmetry breaking with $m_{Z}=91 \mathrm{GeV}$ and $\tan \beta=15$. This choice of parameters is made because it results in $M_{h}$ close to $125 \mathrm{GeV}$ (with some mild dependence on $m_{0}$ that is well within the theoretical and parametric uncertainties). The resulting RG trajectories indicating the attraction to the quasifixed points are shown in Fig. 6 for $\hat{m}_{H_{u}}^{2}, \hat{m}_{H_{d}}^{2}$, and $B$. In comparison to the universal gaugino mass case, the results for $\hat{m}_{H_{u}}^{2}$ at scales at and below $Q=\Lambda$ are considerably smaller, so that there is arguably less fine-tuning involved to obtain electroweak symmetry breaking with the observed $m_{Z}$. For example, for all $m_{0}^{2}<(4 \mathrm{TeV})^{2}$, the value of $\hat{m}_{H_{u}}$ at $Q=\Lambda$ is less than $1 \mathrm{TeV}$, and it maintains a more moderate slope throughout its running, compared to the scenario with unified gaugino masses at the GUT scale.

The running mass of a right-handed squark is shown in the left panel of Fig. 7, along with the gluino running mass parameter $M_{3}$. Both the gluino and squarks can be much lighter than in the universal gaugino mass case and can easily be less than $3 \mathrm{TeV}$ and therefore accessible to the LHC with sufficient integrated luminosity. No effort was made to fine tune the chosen model to be optimized in this regard, so that even somewhat lighter squarks and gluino are possible. As in the universal gaugino mass case, the right-handed squarks are lighter than the gluino, but the left-handed squarks can be heavier than the gluino, due to the RG influence of the much larger value of $M_{2}^{2}$ compared to $M_{3}^{2}$ here. 

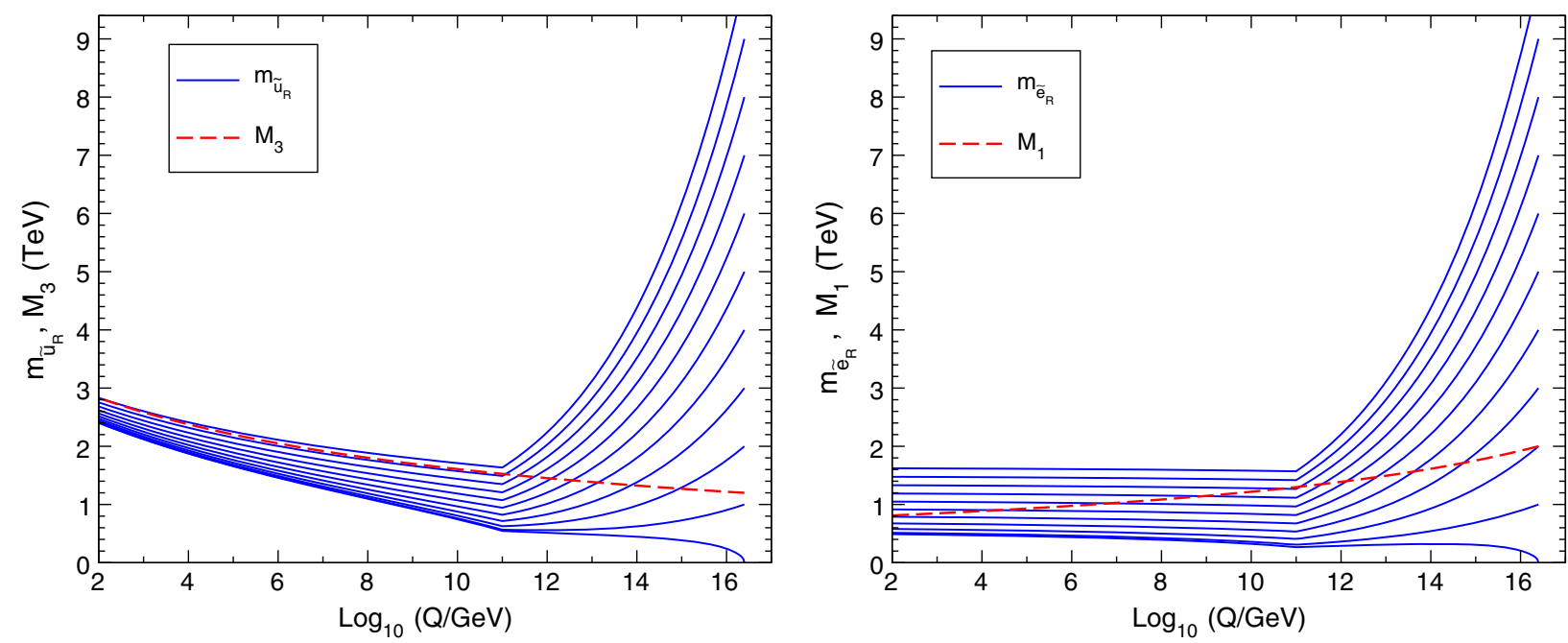

FIG. 7. RG running of the squark and slepton mass parameters $m_{\tilde{u}_{R}}$ (left panel) and $m_{\tilde{e}_{R}}$ (right panel) for a model with nonuniversal gaugino masses $M_{1}=2000 \mathrm{GeV}, M_{2}=4000 \mathrm{GeV}$, and $M_{3}=1200 \mathrm{GeV}$ at the GUT scale. Each line is the RG trajectory for a different boundary condition of the common scalar mass at the GUT scale, showing the approach to the quasifixed point behavior. The running gluino and bino masses $M_{3}$ and $M_{1}$ are also shown for comparison.

The right panel of Fig. 7 shows the running of the righthanded selectron mass compared to the running bino mass $M_{1}$. This is qualitatively quite similar to the situation in the universal gaugino mass case. However, one important difference is that in this case the lighter stau (not shown in Fig. 7) can have a slightly smaller mass than the righthanded selectron and smuon. As before, the LSP is predicted to be the binolike neutralino if $m_{0} \gtrsim 2.5 M_{1}$ at $Q=M_{*} ;$ otherwise, it is a stau, and $R$-parity violation can be invoked to avoid a disastrous stable charged relic from the early Universe.

Figure 8 shows the physical mass spectrum as a function of $m_{0}$. Some salient features of this model line with $M_{3} / M_{2} \sim 0.3$ that are different from the universal gaugino mass case of the previous subsection are as follows:

(i) The superpartner mass spectrum is relatively compressed compared to scenarios based on unified gaugino masses at the GUT scale, but (in this example, at least) not enough to dramatically impact typical LHC search strategies for a given gluino mass,

(ii) The heaviest superpartner is a winolike chargino/ neutralino (or perhaps a left-handed squark if $m_{0}$ is very large), so that decays through (off-shell) winos are suppressed,

(iii) The gluino and squarks are beyond the reach of the collected LHC data as of this writing, but might be seen at a future luminosity or higher-energy upgrade,

(iv) The lightest of the sleptons is a stau, rather than a right-handed selectron or smuon.

\section{OUTLOOK}

In this paper, I have noted that in scalar sequestering models of low-energy supersymmetry, the interplay between the strong superconformal dynamics in the hidden sector and the perturbative renormalization effects of the visible sector results in running toward nontrivial quasifixed point RG trajectories at an intermediate scale. The scalar squared masses of the theory are suppressed but run to values that are non-negligible, because the scaling parameter $\Gamma$ cannot be too large. It is tantalizing that the

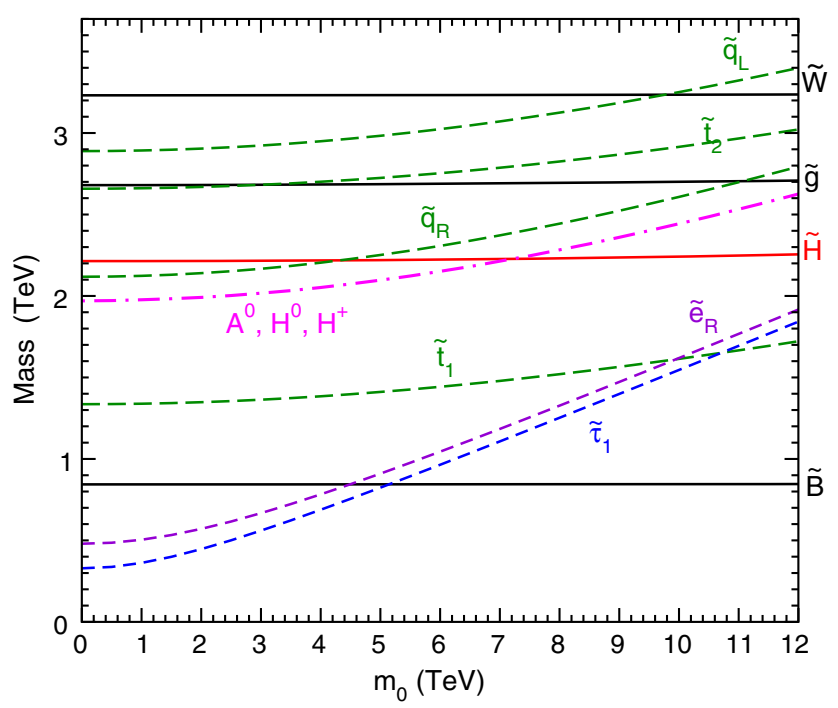

FIG. 8. The spectrum of physical masses of the gluino, squarks, sleptons, electroweakinos, and the heavier Higgs scalar bosons, for a model line with fixed GUT-scale input parameters $M_{3}=1200 \mathrm{GeV}, \quad M_{2}=4000 \mathrm{GeV}, \quad M_{1}=2000 \mathrm{GeV}, \quad$ and $b=(2000 \mathrm{GeV})^{2}$, as a function of $m_{0}$. The scalar trilinear coupling parameter $A_{0}$ and the Higgsino mass parameter $\mu$ are determined by requiring electroweak symmetry breaking with $m_{\mathrm{Z}}=91 \mathrm{GeV}$ and $\tan \beta=15$. The lightest neutral Higgs boson mass is close to $125 \mathrm{GeV}$, due to large top-squark mixing. 
combination $m_{H_{u}}^{2}+|\mu|^{2}$ is subject to this suppression, because this is the combination that should be small at the $\mathrm{TeV}$ scale in order to solve the little hierarchy problem. In the end, taking into account the constraints on anomalous dimensions, it appears that there is some amelioration of the little hierarchy problem, due to the suppression proportional to $3 / 8 \pi^{2} \Gamma$ in Eq. (3.8), especially in models with $M_{3}<M_{2}$ at the high input scale. However, I would not argue that this attains a completely compelling solution to the problem (which is in any case somewhat subjective), as the experimental value of $m_{Z}^{2}$ is still quite small compared to the individual MSSM parameter contributions to it.

Models of this kind do at least have the virtue of providing some predictive power, compared to the general MSSM, due to the renormalization group quasifixed point structure. The superpartner mass spectrum depends only weakly, and in specific calculable ways, on the input values of the dimension-2 parameters. It should be remarked that the details of the scalar sequestering necessarily cannot be predicted with fine precision, given the lack of knowledge of the hidden-sector dynamics. The anomalous dimensions of a putative superconformal theory are bounded but not known, and the hidden sector is likely to be approximately, but not exactly, superconformal. While the predictivity of this model framework is thus limited, general and qualitative statements can still be made. A prominent example of this predictivity is that imposing gaugino mass unification seems to require superpartners definitely beyond the reach of the LHC even after a high-luminosity upgrade, given the observed mass of the lightest Higgs scalar boson at $M_{h}=125 \mathrm{GeV}$. By relaxing the assumption to allow $M_{3}<M_{2}$ at the input scale, I argued that the scenario becomes subjectively more natural, while also allowing for a chance at discovery in the future at the LHC.

\section{ACKNOWLEDGMENTS}

I thank Simon Knapen and Martin Schmaltz for useful conversations. This work was performed in part in the exalted atmosphere of the Aspen Center for Physics, which is supported by National Science Foundation Grant No. PHY-1607611. This work was supported in part by the National Science Foundation Grant No. PHY-1719273.
[1] For a review with conventions and notations consistent with the present paper, see S. P. Martin, A supersymmetry primer, Adv. Ser. Dir. High Energy Phys. 18, 1 (1998).

[2] S. Dimopoulos, K. Howe, and J. March-Russell, Maximally Natural Supersymmetry, Phys. Rev. Lett. 113, 111802 (2014).

[3] T. Cohen, J. Kearney, and M. Luty, Natural supersymmetry without light Higgsinos, Phys. Rev. D 91, 075004 (2015).

[4] A. E. Nelson and T. S. Roy, New Supersoft Supersymmetry Breaking Operators and a Solution to the $\mu$ Problem, Phys. Rev. Lett. 114, 201802 (2015).

[5] S. P. Martin, Nonstandard supersymmetry breaking and Dirac gaugino masses without supersoftness, Phys. Rev. D 92, 035004 (2015).

[6] M. A. Luty and R. Sundrum, Supersymmetry breaking and composite extra dimensions, Phys. Rev. D 65, 066004 (2002).

[7] M. Luty and R. Sundrum, Anomaly mediated supersymmetry breaking in four-dimensions, naturally, Phys. Rev. D 67, 045007 (2003).

[8] M. Dine, P. J. Fox, E. Gorbatov, Y. Shadmi, Y. Shirman, and S. D. Thomas, Visible effects of the hidden sector, Phys. Rev. D 70, 045023 (2004).

[9] M. Ibe, K.-I. Izawa, Y. Nakayama, Y. Shinbara, and T. Yanagida, Conformally sequestered SUSY breaking in vector-like gauge theories, Phys. Rev. D 73, 015004 (2006).

[10] M. Ibe, K.-I. Izawa, Y. Nakayama, Y. Shinbara, and T. Yanagida, More on conformally sequestered SUSY breaking, Phys. Rev. D 73, 035012 (2006).
[11] M. Schmaltz and R. Sundrum, Conformal sequestering simplified, J. High Energy Phys. 11 (2006) 011.

[12] A. G. Cohen, T. S. Roy, and M. Schmaltz, Hidden sector renormalization of MSSM scalar masses, J. High Energy Phys. 02 (2007) 027.

[13] S. Kachru, L. McAllister, and R. Sundrum, Sequestering in string theory, J. High Energy Phys. 10 (2007) 013.

[14] T. S. Roy and M. Schmaltz, Hidden solution to the $\mu / B \mu$ problem in gauge mediation, Phys. Rev. D 77, 095008 (2008).

[15] H. Murayama, Y. Nomura, and D. Poland, More visible effects of the hidden sector, Phys. Rev. D 77, 015005 (2008).

[16] G. Perez, T. S. Roy, and M. Schmaltz, Phenomenology of SUSY with scalar sequestering, Phys. Rev. D 79, 095016 (2009).

[17] Y. Kawamura, T. Kinami, and T. Miura, Superparticle sum rules in the presence of hidden sector dynamics, J. High Energy Phys. 01 (2009) 064.

[18] M. Asano, J. Hisano, T. Okada, and S. Sugiyama, A realistic extension of gauge-mediated SUSY-breaking model with superconformal hidden sector, Phys. Lett. B 673, 146 (2009).

[19] H. D. Kim and J. H. Kim, Higgs Phenomenology of scalar sequestering, J. High Energy Phys. 05 (2009) 040.

[20] N. J. Craig and D. Green, On the phenomenology of strongly coupled hidden sectors, J. High Energy Phys. 09 (2009) 113. 
[21] K. Hanaki and Y. Ookouchi, Light gauginos and conformal sequestering, Phys. Rev. D 83, 125010 (2011).

[22] N. Craig, S. Knapen, and D. Shih, General messenger Higgs mediation, J. High Energy Phys. 08 (2013) 118.

[23] S. Knapen and D. Shih, Higgs Mediation with strong hidden sector dynamics, J. High Energy Phys. 08 (2014) 136.

[24] A.E. Nelson and M. J. Strassler, Suppressing flavor anarchy, J. High Energy Phys. 09 (2000) 030.

[25] A. E. Nelson and M. J. Strassler, Exact results for supersymmetric renormalization and the supersymmetric flavor problem, J. High Energy Phys. 07 (2002) 021.

[26] D. Poland, D. Simmons-Duffin, and A. Vichi, Carving out the space of 4D CFTs, J. High Energy Phys. 05 (2012) 110.

[27] D. Poland and A. Stergiou, Exploring the minimal 4D $\mathcal{N}=1$ SCFT, J. High Energy Phys. 12 (2015) 121.

[28] D. Green and D. Shih, Bounds on SCFTs from conformal perturbation theory, J. High Energy Phys. 09 (2012) 026.

[29] S. P. Martin and M. T. Vaughn, Two loop renormalization group equations for soft supersymmetry breaking couplings, Phys. Rev. D 50, 2282 (1994); Errattum, Phys. Rev. D 78, 039903(E) (2008).

[30] Y. Yamada, Two loop renormalization group equations for soft SUSY breaking scalar interactions: Supergraph method, Phys. Rev. D 50, 3537 (1994).

[31] I. Jack and D. R. T. Jones, Soft supersymmetry breaking and finiteness, Phys. Lett. B 333, 372 (1994).

[32] I. Jack, D. R. T. Jones, S. P. Martin, M. T. Vaughn, and Y. Yamada, Decoupling of the epsilon scalar mass in softly broken supersymmetry, Phys. Rev. D 50, R5481 (1994).

[33] L. Randall and R. Sundrum, Out of this world supersymmetry breaking, Nucl. Phys. B557, 79 (1999); G. F. Giudice, M. A. Luty, H. Murayama, and R. Rattazzi, Gaugino mass without singlets, J. High Energy Phys. 12 (1998) 027.

[34] S. P. Martin, Three-loop corrections to the lightest Higgs scalar boson mass in supersymmetry, Phys. Rev. D 75, 055005 (2007).

[35] For an incomplete list of recent discussions from various points of view, see the following, and references therein: P. Draper, G. Lee, and C. E. M. Wagner, Precise estimates of the Higgs mass in heavy supersymmetry, Phys. Rev. D 89, 055023 (2014); J. Pardo Vega and G. Villadoro, SusyHD: Higgs mass determination in supersymmetry, J. High Energy Phys. 07 (2015) 159; T. Hahn, S. Heinemeyer, W. Hollik, H. Rzehak, and G. Weiglein, High precision prediction for $M_{h}$ in the MSSM, Nucl. Part. Phys. Proc. 273-275, 794 (2016); P. Athron, J. h. Park, T. Steudtner, D. Stöckinger, and A. Voigt, Precise Higgs mass calculations in (non)minimal supersymmetry at both high and low scales, J. High Energy Phys. 01 (2017) 079; F. Staub and W. Porod, Improved predictions for intermediate and heavy Supersymmetry in the MSSM and beyond, Eur. Phys. J. C 77, 338 (2017); E. Bagnaschi, J. Pardo Vega, and P. Slavich, Improved determination of the Higgs mass in the MSSM with heavy superpartners, Eur. Phys. J. C 77, 334 (2017); H. Bahl, S. Heinemeyer, W. Hollik, and G. Weiglein, Reconciling EFT and hybrid calculations of the light MSSM Higgs-boson mass, arXiv:1706.00346; R. V. Harlander, J. Klappert, and A. Voigt, Higgs mass prediction in the MSSM at three-loop level in a pure DR context, Eur. Phys. J. C 77, 814 (2017).
[36] J. R. Ellis, C. Kounnas, and D. V. Nanopoulos, No scale supersymmetric GUTs, Nucl. Phys. B247, 373 (1984).

[37] J. R. Ellis, A. B. Lahanas, D. V. Nanopoulos, and K. Tamvakis, No-scale supersymmetric standard model, Phys. Lett. 134B, 429 (1984).

[38] A. B. Lahanas and D. V. Nanopoulos, The road to no scale supergravity, Phys. Rep. 145, 1 (1987).

[39] D. E. Kaplan, G. D. Kribs, and M. Schmaltz, Supersymmetry breaking through transparent extra dimensions, Phys. Rev. D 62, 035010 (2000).

[40] Z. Chacko, M. A. Luty, A. E. Nelson, and E. Ponton, Gaugino mediated supersymmetry breaking, J. High Energy Phys. 01 (2000) 003.

[41] M. Schmaltz and W. Skiba, Minimal gaugino mediation, Phys. Rev. D 62, 095005 (2000).

[42] J. R. Ellis, K. Enqvist, D. V. Nanopoulos, and K. Tamvakis, Gaugino masses and grand unification, Phys. Lett. B 155, 381 (1985).

[43] M. Drees, Phenomenological consequences of $N=1$ supergravity theories with nonminimal kinetic-energy terms for vector superfields, Phys. Lett. 158B, 409 (1985).

[44] G. Anderson, C. H. Chen, J. F. Gunion, J. D. Lykken, T. Moroi, and Y. Yamada, Motivations for and implications of non-universal GUT-scale boundary conditions for soft SUSY-breaking parameters, eConf C960625, SUP107 (1996).

[45] G. Anderson, H. Baer, C. h. Chen, and X. Tata, The reach of Fermilab Tevatron upgrades for SU(5) supergravity models with non-universal gaugino masses, Phys. Rev. D 61, 095005 (2000).

[46] J. Chakrabortty and A. Raychaudhuri, A note on dimension5 operators in GUTs and their impact, Phys. Lett. B 673, 57 (2009).

[47] S.P. Martin, Non-universal gaugino masses from nonsinglet F-terms in non-minimal unified models, Phys. Rev. D 79, 095019 (2009).

[48] G. L. Kane and S. F. King, Naturalness implications of LEP results, Phys. Lett. B 451, 113 (1999); M. Bastero-Gil, G. L. Kane, and S.F. King, Fine-tuning constraints on supergravity models, Phys. Lett. B 474, 103 (2000).

[49] H. Baer, A. Mustafayev, E.-K. Park, S. Profumo, and X. Tata, Mixed Higgsino dark matter from a reduced SU(3) gaugino mass: Consequences for dark matter and collider searches, J. High Energy Phys. 04 (2006) 041.

[50] H. Abe, T. Kobayashi, and Y. Omura, Relaxed fine-tuning in models with non-universal gaugino masses, Phys. Rev. D 76, 015002 (2007).

[51] S. P. Martin, Compressed supersymmetry and natural neutralino dark matter from top squark-mediated annihilation to top quarks, Phys. Rev. D 75, 115005 (2007).

[52] H. Baer, A. Mustafayev, H. Summy, and X. Tata, Mixed Higgsino dark matter from a large SU(2) gaugino mass, J. High Energy Phys. 10 (2007) 088.

[53] D. Horton and G. G. Ross, Naturalness and focus points with non-universal gaugino masses, Nucl. Phys. B830, 221 (2010).

[54] J.E. Younkin and S.P. Martin, Non-universal gaugino masses, the supersymmetric little hierarchy problem, and dark matter, Phys. Rev. D 85, 055028 (2012); S. P. Martin, Nonuniversal gaugino masses and seminatural 
supersymmetry in view of the Higgs boson discovery, Phys. Rev. D 89, 035011 (2014).

[55] F. Brummer and W. Buchmuller, The Fermi scale as a focus point of high-scale gauge mediation, J. High Energy Phys. 05 (2012) 006.

[56] S. Antusch, L. Calibbi, V. Maurer, M. Monaco, and M. Spinrath, Naturalness of the non-universal MSSM in the light of the recent Higgs results, J. High Energy Phys. 01 (2013) 187.

[57] I. Gogoladze, F. Nasir, and Q. Shafi, Non-universal gaugino masses and natural supersymmetry, Int. J. Mod. Phys. A 28, 1350046 (2013).

[58] A. Spies and G. Anton, Confronting recent results from selected direct and indirect dark matter searches and the Higgs boson with supersymmetric models with non-universal gaugino masses, J. Cosmol. Astropart. Phys. 06 (2013) 022.

[59] T. T. Yanagida and N. Yokozaki, Focus point in gaugino mediation reconsideration of the fine-tuning problem,
Phys. Lett. B 722, 355 (2013); Bino-Higgsino mixed dark matter in a focus point gaugino mediation, J. High Energy Phys. 11 (2013) 020.

[60] K. L. Chan, U. Chattopadhyay, and P. Nath, Naturalness, weak scale supersymmetry and the prospect for the observation of supersymmetry at the Tevatron and at the LHC, Phys. Rev. D 58, 096004 (1998); J. L. Feng, K. T. Matchev, and T. Moroi, Multi-TeV Scalars are Natural in Minimal Supergravity, Phys. Rev. Lett. 84, 2322 (2000); Focus points and naturalness in supersymmetry, Phys. Rev. D 61, 075005 (2000); J. L. Feng, K. T. Matchev, and F. Wilczek, Neutralino dark matter in focus point supersymmetry, Phys. Lett. B 482, 388 (2000); J. L. Feng, K. T. Matchev, and D. Sanford, Focus point supersymmetry redux, Phys. Rev. D 85, 075007 (2012); J. L. Feng and D. Sanford, A natural $125 \mathrm{GeV}$ Higgs boson in the MSSM from focus point supersymmetry with A-terms, Phys. Rev. D 86, 055015 (2012). 